\begin{tabular}{|c|l|}
\hline Title & Low-temperature thermodynamics of one dimensional alternating-spin Heisenberg ferromagnets \\
\hline Author(s) & Y amamoto, Shoji; Hori, Hiromitsu \\
\hline Citation & $\begin{array}{l}\text { PHY SICAL REVIEW B, 72, 054423 } \\
\text { https://doi.org/10.1103/PhysRevB.72.054423 }\end{array}$ \\
\hline Issue Date & 2005 \\
\hline Doc URL & http://hdl.handle.net/2115/5862 \\
\hline Rights & Copyright $\odot 2005$ A merican Physical Society \\
\hline Type & article \\
\hline File Information & PRB72.pdf \\
\hline
\end{tabular}

Instructions for use 


\title{
Low-temperature thermodynamics of one-dimensional alternating-spin Heisenberg ferromagnets
}

\author{
Shoji Yamamoto and Hiromitsu Hori \\ Division of Physics, Hokkaido University, Sapporo 060-0810, Japan \\ (Received 27 April 2005; revised manuscript received 20 June 2005; published 17 August 2005)
}

\begin{abstract}
Motivated by a novel bimetallic chain compound in which alternating magnetic centers are ferromagnetically coupled, we investigate thermodynamic properties of one-dimensional spin- $(S, s)$ Heisenberg ferromagnets both numerically and analytically. On the one hand, quantum Monte Carlo calculations illuminate the overall thermal behavior. The specific heat may exhibit a double-peaked structure at intermediate temperatures for $S \gtrsim 3 s$ in general. On the other hand, a modified spin-wave theory precisely describes the low-temperature properties. Expanding the specific heat and the magnetic susceptibility, we reveal an analogy and a contrast between mixed-spin ferromagnets and ferrimagnets.
\end{abstract}

DOI: 10.1103/PhysRevB.72.054423

PACS number(s): 75.10.Jm, 75.40.Cx, 75.30.Ds, 75.40.Mg

\section{INTRODUCTION}

Much effort has been devoted to designing molecular systems ordering ferromagnetically. ${ }^{1}$ One possible approach consists of assembling molecular bricks so as to obtain a low-dimensional system with a nonzero resultant spin in the ground state and then coupling the chains or the layers again in a ferromagnetic fashion. Numerous heterospin chain compounds have been synthesized along this line. Gleizes and Verdaguer $^{2}$ made an attempt to alternate two types of metal ion along one crystallographic axis with antiferromagnetic intrachain interaction and obtained a pioneering example of quasi-one-dimensional ferrimagnets, of formula $\mathrm{Mn} A \mathrm{Cu}(\text { dto })_{2}\left(\mathrm{H}_{2} \mathrm{O}\right)_{3} \cdot 4.5 \mathrm{H}_{2} \mathrm{O} \quad(A=\mathrm{Ni}, \mathrm{Cu} ; \quad$ dto = dithiooxalato $=\mathrm{S}_{2} \mathrm{C}_{2} \mathrm{O}_{2}$ ). Kahn et al. ${ }^{3}$ synthesized another series of bimetallic chain compounds $A \mathrm{Cu}(\mathrm{pbaOH})$ $\times\left(\mathrm{H}_{2} \mathrm{O}\right)_{3} \cdot n \mathrm{H}_{2} \mathrm{O} \quad[A=\mathrm{Fe}, \mathrm{Co}, \mathrm{Ni}, \mathrm{Cu} ; \quad$ pbaOH $=2$-hydroxys -1,3-propylenebis (oxamato) $\left.=\mathrm{C}_{7} \mathrm{H}_{6} \mathrm{~N}_{2} \mathrm{O}_{7}\right]$, one of which indeed attained the three-dimensional ferromagnetic order at low temperatures. ${ }^{4}$ Caneschi et al. ${ }^{5}$ took an alternative strategy of bringing into interaction metal ions and stable organic radicals. This idea was developed toward polymeric chain compounds. ${ }^{6}$ The wide variety of chemical explorations stimulated the physical interest in mixed-spin chains. ${ }^{7-15}$

Most of the thus-far synthesized heterospin systems are characterized as ferrimagnets. Ferromagnetic intrachain coupling is observed in few cases. In such circumstances, $\mathrm{MnNi}\left(\mathrm{NO}_{2}\right)_{4}(\mathrm{en})_{2}\left(\mathrm{en}=\right.$ ethylenediamine $\left.=\mathrm{C}_{2} \mathrm{H}_{8} \mathrm{~N}_{2}\right),{ }^{16}$ proved to be a quasi-one-dimensional mixed-spin ferromagnet ${ }^{17}$ and caused renewed interest in mixed-spin chains. Gillon et al. ${ }^{18}$ calculated the spin density distribution by means of the density functional theory and quantitatively visualized the ferromagnetic nature of the $\mathrm{Mn}(\mathrm{II})-\mathrm{Ni}(\mathrm{II})$ interaction. Fukushima et al. ${ }^{19}$ performed high-temperature series expansion of the thermal quantities and argued the magnetic structure including single-ion anisotropy and interchain exchange coupling. Now an increasing number of chemists and physicists are taking interest in heterospin ferromagnets. ${ }^{20,21}$

Alternating-spin chains possess elementary excitations of dual aspect. In the case of antiferromagnetic coupling, the acoustic excitations reduce the ground-state magnetization and are thus of ferromagnetic nature, while the optical exci- tations enhance the ground-state magnetization and are thus of antiferromagnetic nature. In the case of ferromagnetic coupling, on the other hand, both excitations are of ferromagnetic character. Therefore, the Schottky-type peak of the specific heat and the minimum of the susceptibilitytemperature product, which are both ferrimagnetic features, ${ }^{10,22}$ are absent from mixed-spin ferromagnets. Nevertheless, mixed-spin ferromagnets and ferrimagnets behave similarly at low temperatures, which is the goal of this paper. Employing the world-line quantum Monte Carlo method ${ }^{23}$ and a modified spin-wave theory, ${ }^{24}$ we investigate thermodynamics of one-dimensional alternating-spin Heisenberg ferromagnets with particular emphasis on the intrinsic lowtemperature properties.

\section{MODIFIED SPIN-WAVE SCHEME}

We consider two kinds of spins $S$ and $s(S>s)$ alternating on a ring with ferromagnetic exchange coupling $(J>0)$ between nearest neighbors, as described by the Hamiltonian

$$
\mathcal{H}=-J \sum_{n=1}^{N}\left(\boldsymbol{S}_{n} \cdot \boldsymbol{s}_{n}+\boldsymbol{s}_{n} \cdot \boldsymbol{S}_{n+1}\right)
$$

Even in one dimension, the conventional spin-wave theory ${ }^{25-27}$ gives a fine piece of information on the groundstate correlation. ${ }^{28,29}$ As for the thermal quantities, however, the low-temperature series expansion within the conventional scheme $\mathrm{e}^{30}$ only reproduces the leading term of the specific heat and nothing correct for the magnetic susceptibility. ${ }^{31}$ Then Takahashi ${ }^{32}$ modified the spin-wave formalism, imposing a constraint on the magnetization, and obtained an excellent description of the low-temperature thermodynamics of low-dimensional ferromagnets. We develop the modified scheme for mixed-spin ferromagnets.

In order to describe the spin deviation in each sublattice, bosonic operators are introduced as

$$
S_{n}^{+}=\sqrt{2 S-a_{n}^{\dagger} a_{n}} a_{n}, \quad S_{n}^{z}=S-a_{n}^{\dagger} a_{n},
$$


(a) $(1,1 / 2)$

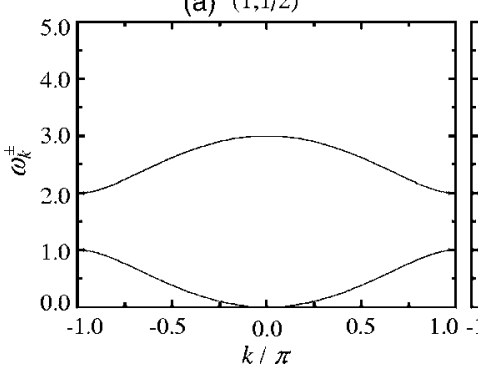

$s_{n}^{+}=\sqrt{2 s-b_{n}^{\dagger} b_{n}} b_{n}, \quad s_{n}^{z}=s-b_{n}^{\dagger} b_{n}$,

where we regard $S$ and $s$ as quantities of the same order. The bosonic Hamiltonian reads

$$
\mathcal{H}=E_{2}+\mathcal{H}_{1}+\mathcal{H}_{0}+O\left(S^{-1}\right),
$$

where $E_{2}=-2 S S J N$ is the classical ground-state energy and $\mathcal{H}_{i}$ is the $O\left(S^{i}\right)$ quantum correction to it. We consider first diagonalizing $\mathcal{H}_{1}$ and then taking $\mathcal{H}_{0}$ into calculation perturbationally. ${ }^{33}$ Via the transformation

$$
\begin{aligned}
& a_{n}^{\dagger}=\frac{1}{\sqrt{N}} \sum_{k} e^{-\mathrm{i} k(n-1 / 4)}\left(\alpha_{k}^{\dagger} \cos \theta_{k}-\beta_{k}^{\dagger} \sin \theta_{k}\right), \\
& b_{n}^{\dagger}=\frac{1}{\sqrt{N}} \sum_{k} e^{-\mathrm{i} k(n+1 / 4)}\left(\alpha_{k}^{\dagger} \sin \theta_{k}+\beta_{k}^{\dagger} \cos \theta_{k}\right),
\end{aligned}
$$

with $\tan \left(2 \theta_{k}\right)=2 \sqrt{S s} \cos (k / 2) /(S-s)$, we obtain

$$
\mathcal{H}_{1}=J \sum_{k}\left(\omega_{k}^{-} \alpha_{k}^{\dagger} \alpha_{k}+\omega_{k}^{+} \beta_{k}^{\dagger} \beta_{k}\right)
$$

Here the acoustic $\left(\omega_{k}^{-}\right)$and optical $\left(\omega_{k}^{+}\right)$dispersion relations are given by

$$
\omega_{k}^{ \pm}=S+s \pm \sqrt{(S-s)^{2}+4 S s \cos ^{2}(k / 2)} \equiv S+s \pm \omega_{k},
$$

and plotted in Fig. 1.

Now we proceed to the modified spin-wave scheme in an attempt to avoid thermal divergence of the number of bosons. At finite temperatures, we replace $\alpha_{k}^{\dagger} \alpha_{k}$ and $\beta_{k}^{\dagger} \beta_{k}$ by $\bar{n}_{k}^{\overline{+}} \equiv \sum_{n^{-}, n^{+}} n^{\overline{+}} P_{k}\left(n^{-}, n^{+}\right)$, where $P_{k}\left(n^{-}, n^{+}\right)$is the probability of $n^{-}$acoustic and $n^{+}$optical spin waves appearing in the $k$-momentum state, and minimize the up-to- $O\left(S^{1}\right)$ free energy

$$
\mathcal{F}=E_{2}+J \sum_{k} \sum_{\sigma= \pm} \omega_{k}^{\sigma} \bar{n}_{k}^{\sigma}+k_{B} T \sum_{k} \sum_{n^{-}, n^{+}} P_{k}\left(n^{-}, n^{+}\right) \ln P_{k}\left(n^{-}, n^{+}\right),
$$

with respect to $P_{k}\left(n^{-}, n^{+}\right)$'s under the condition of zero magnetization

$$
(S+s) N-\sum_{k} \sum_{\sigma= \pm} \bar{n}_{k}^{\sigma}=0
$$

together with the trivial constraints $\sum_{n^{-}, n^{+}} P_{k}\left(n^{-}, n^{+}\right)=1$. Up to $O\left(S^{1}\right)$, the magnetic susceptibility and the internal energy at thermal equilibrium are expressed as
FIG. 1. Single-magnon excitation spectra as the rigorous dispersion relations of the elementary excitations for the spin- $(S, s)$ ferromagnetic Heisenberg chains.

with $\bar{n}_{k}^{ \pm}=\left[e^{\left(J \omega_{k}^{ \pm}-\mu\right) / k_{B} T}-1\right]^{-1}$, where the $g$ factors of the spins $S$ and $s$ are both set equal to $g$ and the Lagrange multiplier $\mu$ is determined through the condition (2.8). The specific heat $C$ is calculated by numerically differentiating the internal energy. The perturbational correction of $O\left(S^{0}\right)$ reads

$$
\begin{aligned}
\left\langle H_{0}\right\rangle \equiv & \operatorname{Tr}\left[\mathcal{H}_{0} e^{-\mathcal{H}_{1} / k_{B} T}\right] / \operatorname{Tr}\left[e^{-\mathcal{H}_{1} / k_{B} T}\right] \\
= & \frac{J N}{2}\left[\sqrt{\frac{S}{S}}\left(\Gamma_{1}-\Gamma_{2}\right) \Gamma_{3}+\sqrt{\frac{s}{S}}\left(\Gamma_{1}+\Gamma_{2}\right) \Gamma_{3}\right. \\
& \left.-\Gamma_{1}^{2}+\Gamma_{2}^{2}-\Gamma_{3}^{2}\right],
\end{aligned}
$$

with

$$
\begin{gathered}
\Gamma_{1}=\frac{1}{N} \sum_{k}\left(\bar{n}_{k}^{-}+\bar{n}_{k}^{+}\right)=S+s, \\
\Gamma_{2}=\frac{1}{N} \sum_{k} \frac{S-s}{\omega_{k}}\left(\bar{n}_{k}^{-}-\bar{n}_{k}^{+}\right),
\end{gathered}
$$

$$
\Gamma_{3}=\frac{1}{N} \sum_{k} \frac{2 \sqrt{S s}}{\omega_{k}} \cos ^{2} \frac{k}{2}\left(\bar{n}_{k}^{-}-\bar{n}_{k}^{+}\right)
$$

where we keep $\mu$ unchanged. Indeed $\mu$ may be modified so as to minimize the up-to- $O\left(S^{0}\right)$ free energy, but such a procedure, which is much more complicated, has no effect on the low-temperature leading behavior. ${ }^{30}$ The thermal quantities are expanded in powers of $\left(k_{B} T / J\right)^{1 / 2}$ at low temperatures. The specific heat and the magnetic susceptibility start from $T^{1 / 2}$ and $T^{-2}$, respectively, and their leading three terms are not affected by the $O\left(S^{0}\right)$ interactions.

\section{NUMERICAL RESULTS FOR THE OVERALL THERMAL BEHAVIOR}

The world-line quantum Monte Carlo calculation is carried out at $N=24,32,40(48,64,80$ spins $)$ and is extrapolated to the $N \rightarrow \infty$ limit. However, any quantity divided by $N$ does not vary with $N$ beyond its statistical error in the temperature 


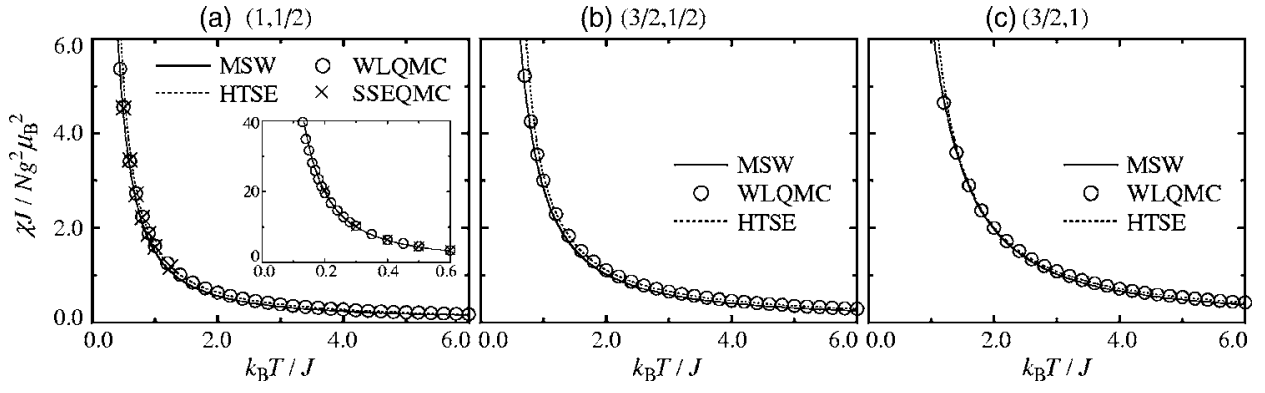

FIG. 2. The modified-spin-wave (MSW) and the world-line quantum Monte Carlo (WLQMC) calculations of the magnetic susceptibility $\chi$ as a function of temperature for the spin- $(S, s)$ ferromagnetic Heisenberg chains. The numerical error is within the symbol size. Hightemperature series-expansion (HTSE) and stochastic series-expansion quantum Monte Carlo (SSEQMC) calculations, which were performed up to $O\left[\left(J / k_{B} T\right)^{7}\right]$ and at $N=64$, respectively, by Fukushima et al. (Refs. 19 and 34), are also shown for reference. At $(S, s)=\left(1, \frac{1}{2}\right)$, the low-temperature behavior is scaled up in an inset.

range to show. A few million Monte Carlo steps are spent on low-temperature calculations, while less than a half million steps on high-temperature calculations. The numerical precision in the final results is two to three digits.

In Fig. 2 we show the modified-spin-wave and the worldline quantum Monte Carlo calculations of the magnetic susceptibility together with preceding findings ${ }^{19,34}$ through the power series expansion of $e^{-\mathcal{H} / k_{B} T}$. Fukushima et al. pioneeringly applied a quantum Monte Carlo scheme combined with the stochastic series-expansion technique ${ }^{35}$ to the mixed-spin ferromagnetic thermodynamics and further performed hightemperature series expansion for general $S$ and $s$. The modified spin-wave calculations are in good agreement with numerical findings over the whole temperature range, reproducing the paramagnetic susceptibility $[S(S+1)+s(s$ $+1)] N\left(g \mu_{B}\right)^{2} / 3 k_{B} T$ at high temperatures and revealing the $T^{-2}$-diverging behavior at low temperatures. The susceptibility-temperature product still monotonically decreases with increasing temperature in contrast with the ferrimagnetic behavior. ${ }^{36}$ The low-temperature behavior is later discussed in more detail.

In Fig. 3 we show the modified spin-wave and the worldline quantum Monte Carlo calculations of the specific heat together with preceding findings. ${ }^{19,34}$ The modified spinwave calculations are less precise than those for the susceptibility but well reproduce the $T^{1 / 2}$-initial behavior at low temperatures and the spin-dependent peak structure at intermediate temperatures. The midtemperature structure of the specific heat may be regarded as a function of the acoustic excitation band width $W^{-}=2 s J$ and the optical excitation gap $\Delta=2 S J$ (see Fig. 1). The heat capacity attributable to the acoustic excitations and that to the optical excitations may be separable when $W^{-} \ll \Delta$. We find a single peak in Fig. 3(c) with $S / s=3 / 2$ but two humps in Fig. 3(b) with $S / s=3$. Further calculations for higher spins (Fig. 4) suggest that the peak stays single at $S / s=2$, while it splits in two at $S / s=4$. The intermediate temperature dependence is more featured with increasing $S / s$. We are empirically convinced that the double-peaked structure may appear for $S \gtrsim 3 s$, including practical cases $(S, s)=\left(\frac{5}{2}, \frac{1}{2}\right),\left(2, \frac{1}{2}\right),\left(\frac{3}{2}, \frac{1}{2}\right) . \quad \mathrm{Mn}(\mathrm{II}) \mathrm{Cu}(\mathrm{II})$, $\mathrm{Fe}(\mathrm{II}) \mathrm{Cu}(\mathrm{II})$, and $\mathrm{Co}(\mathrm{II}) \mathrm{Cu}(\mathrm{II})$ chain compounds ${ }^{3}$ have indeed been synthesized so far, but they all exhibit antiferromagnetic intrachain interaction. The double-peaked structure is much more pronounced for ferromagnetic intrachain interaction. ${ }^{19,34,37}$ We expect an increased effort to design ferromagnetic exchange coupling between alternating metal ions.

\section{ANALYTICAL RESULTS FOR THE LOW- TEMPERATURE BEHAVIOR}

In order to elucidate the low-temperature thermal behavior, we define the state density function

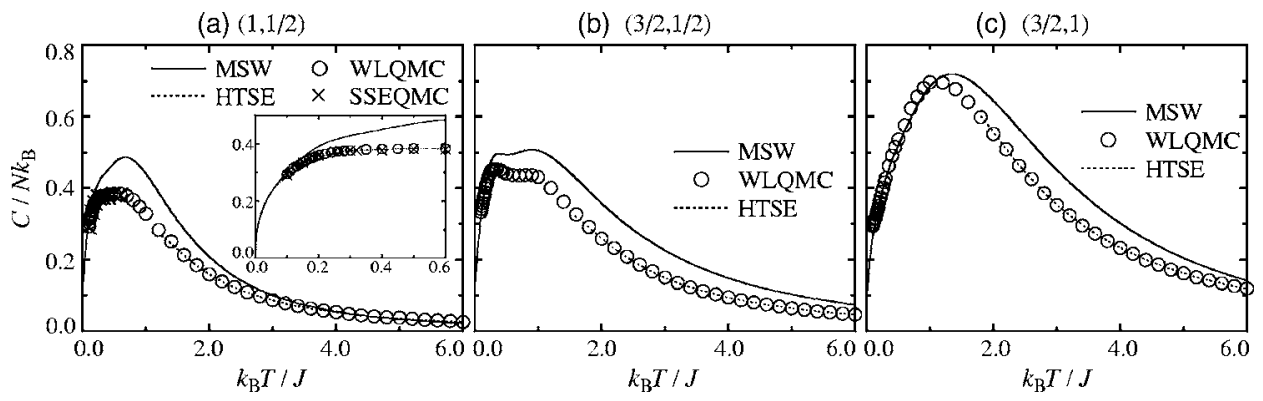

FIG. 3. The modified-spin-wave (MSW) and the world-line quantum Monte Carlo (WLQMC) calculations of the specific heat $C$ as a function of temperature for the spin- $(S, s)$ ferromagnetic Heisenberg chains. The numerical error is within the symbol size. High-temperature series-expansion (HTSE) and stochastic series-expansion quantum Monte Carlo (SSEQMC) calculations, which were performed up to $O\left[\left(J / k_{B} T\right)^{11}\right]$ and at $N=50$, respectively, by Fukushima et al. (Refs. 19 and 34), are also shown for reference. At $(S, s)=\left(1, \frac{1}{2}\right)$, the low-temperature behavior is scaled up in an inset, where HTSE findings were elaborately obtained through a sophisticated Padé analysis (Ref. 19) of the series up to $O\left[\left(J / k_{B} T\right)^{29}\right]$. 
(a) $(2,1)$

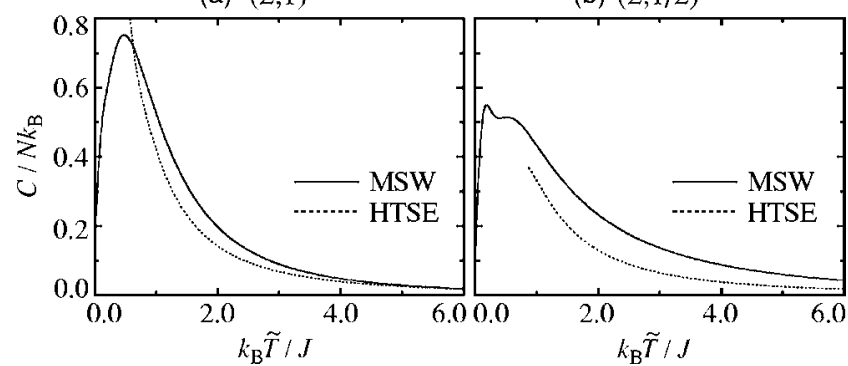

FIG. 4. The modified-spin-wave (MSW) calculations of the specific heat $C$ as a function of temperature for the spin- $(S, s)$ ferromagnetic Heisenberg chains in the cases of $S<3 s$ (a) and $S>3 s$ (b). High-temperature series-expansion (HTSE) calculations up to $O\left[\left(J / k_{B} T\right)^{11}\right]$ by Fukushima et al. (Ref. 19) are also shown for reference. The temperature scale is effectively enhanced with increasing $S$ and $s$ and therefore temperature is renormalized as $T / \sqrt{S s(S+1)(s+1)} \equiv \widetilde{T}$ (Ref. 19).

$$
w^{ \pm}(x)=\frac{1}{2 \pi} \int_{-\pi}^{\pi} \delta\left(x-\omega_{k}^{ \pm}\right) d k
$$

Here we are interested in the gapless acoustic branch and expand $w^{-}(x)$ for small $x$ as

$$
w^{-}(x)=\frac{1}{\pi} \sqrt{\frac{S+s}{2 S s x}} \sum_{n=0}^{\infty} c_{n}^{-} x^{n} .
$$

A few leading coefficients are given as

$$
\begin{gathered}
c_{0}^{-}=1, \\
c_{1}^{-}=\frac{(S-s)^{2}+S s}{4 S s(S+s)}, \\
c_{2}^{-}=\frac{\left(3 S^{2}-4 S s+3 s^{2}\right)(S+s)^{2}-5 S^{2} s^{2}}{32 S^{2} s^{2}(S+s)^{2}} .
\end{gathered}
$$

Applying Eq. (4.2) and neglecting the optical excitations $\bar{n}_{k}^{+}$, Eq. (2.8) reads

$$
\begin{aligned}
v^{1 / 2}= & \frac{1}{\pi \sqrt{2 S s(S+s)}} \sum_{n=0}^{\infty} c_{n}^{-} t^{n+1 / 2} \Gamma\left(n+\frac{1}{2}\right)\left[\Gamma\left(\frac{1}{2}-n\right) v^{n}\right. \\
& \left.+\sum_{m=0}^{\infty} \frac{(-1)^{m}}{m !} \zeta\left(n-m+\frac{1}{2}\right) v^{m+1 / 2}\right]
\end{aligned}
$$

where $v=-\mu / k_{B} T, t=k_{B} T / J$, and $\zeta(n)$ is Riemann's zeta function. Solving this equation iteratively, we obtain the lowtemperature expansion of the Lagrange multiplier as

$$
\begin{aligned}
v^{1 / 2}= & \frac{c_{0}^{-} \Gamma(1 / 2)}{\pi \sqrt{2 S s(S+s)}} \Gamma\left(\frac{1}{2}\right) t^{1 / 2} \\
& +\left[\frac{c_{0}^{-} \Gamma(1 / 2)}{\pi \sqrt{2 S s(S+s)}}\right]^{2} \Gamma\left(\frac{1}{2}\right) \zeta\left(\frac{1}{2}\right) t \\
& +\left[\frac{c_{0}^{-} \Gamma(1 / 2)}{\pi \sqrt{2 S s(S+s)}}\right]^{3} \Gamma\left(\frac{1}{2}\right)\left[\zeta\left(\frac{1}{2}\right)\right]^{2} t^{3 / 2}+O\left(t^{2}\right) .
\end{aligned}
$$

Since the magnetic susceptibility and the internal energy read

$$
\begin{aligned}
\frac{\chi k_{B} T}{N\left(g \mu_{B}\right)^{2}}= & \frac{1}{3 \pi} \sqrt{\frac{S+s}{2 S s}} \sum_{n=0}^{\infty} c_{n}^{-} t^{n+1 / 2} \Gamma\left(n+\frac{1}{2}\right)\left[\Gamma\left(\frac{3}{2}-n\right) v^{n-3 / 2}\right. \\
& \left.+\sum_{m=0}^{\infty} \frac{(-1)^{m}}{m !} \zeta\left(n-m-\frac{1}{2}\right) v^{m}\right], \\
\frac{E-E_{2}}{N J}= & \frac{1}{\pi} \sqrt{\frac{S+s}{2 S s} \sum_{n=0}^{\infty} c_{n}^{-} t^{n+3 / 2} \Gamma\left(n+\frac{3}{2}\right)\left[\Gamma\left(-\frac{1}{2}-n\right) v^{n+1 / 2}\right.} \\
& \left.+\sum_{m=0}^{\infty} \frac{(-1)^{m}}{m !} \zeta\left(n-m+\frac{3}{2}\right) v^{m}\right],
\end{aligned}
$$

the susceptibility and the specific heat are expanded as

$$
\begin{aligned}
\frac{\chi J}{N\left(g \mu_{B}\right)^{2}}= & \frac{1}{S s}\left\{\frac{\tilde{t}^{-2}}{3}-\frac{\zeta(1 / 2)}{\sqrt{2 \pi}} \tilde{t}^{-3 / 2}+\left[\frac{\zeta(1 / 2)}{\sqrt{2 \pi}}\right]^{2} \tilde{t}^{-1}\right\} \\
& +O\left(\tilde{t}^{-1 / 2}\right), \\
\frac{C}{N k_{B}}=(S+s)\left\{\frac{3 \zeta(3 / 2)}{4 \sqrt{2 \pi}} \widetilde{t}^{1 / 2}-\tilde{t}\right. & \\
+ & \left.\frac{15\left[\left(S^{2}-S s+s^{2}\right) \zeta(5 / 2)-4 \zeta(1 / 2)\right]}{32 \sqrt{2 \pi}} \widetilde{t}^{3 / 2}\right\}+O\left(\tilde{t}^{2}\right),
\end{aligned}
$$

where $\tilde{t}=t / S s(S+s)=k_{B} T / J S s(S+s)$. The $O\left(S^{0}\right)$ interactions affect the fourth and higher terms and therefore, whether through the Holstein-Primakoff transformation ${ }^{26}$ or through the Dyson-Maleev transformation, ${ }^{27,38}$ we reach the same results (4.8) and (4.9).

Numerically solving the thermodynamic Bethe-ansatz integral equations for the spin- $\frac{1}{2}$ ferromagnetic Heisenberg chain, Takahashi and Yamada ${ }^{31}$ obtained

$$
\begin{gathered}
\frac{\chi J}{L\left(g \mu_{B}\right)^{2}}=0.04167 t^{-2}+0.145 t^{-3 / 2}+0.17 t^{-1}+O\left(t^{-1 / 2}\right) \\
\frac{C}{L k_{B}}=0.7815 t^{1 / 2}-2.00 t+3.5 t^{3 / 2}+O\left(t^{2}\right)
\end{gathered}
$$

where $L$ is the number of spins. When we set $S$ and $s$ both equal to $\frac{1}{2}$, the expressions (4.8) and (4.10) coincide in their leading three terms, while Eqs. (4.9) and (4.11) in their leading two terms. The modified spin-wave calculations are thus 
reliable and give rigorous information on the lowtemperature properties. In the case of arbitrary $S$ and $s$, the leading three terms of Eq. (4.8) and the leading two terms of Eq. (4.9) coincide with those of the spin- $[S s(S+s) / 2]^{1 / 3}$ uniform ferromagnetic chain except for a common factor. Considering practical combinations of $S$ and $s$, we may estimate that $[S s(S+s) / 2]^{1 / 3}=\left[1-(S-s)^{2} /(S+s)^{2}\right]^{1 / 3}(S+s) / 2 \simeq(S$ $+s) / 2$. Thus, ferromagnetically coupled alternating spins $S$ and $s$ look similar to a ferromagnetic assembly of virtual spins $(S+s) / 2$ at low temperatures.

It is also interesting to compare the expressions (4.8) and (4.9) with those of ferrimagnetic chains. ${ }^{24}$ It turns out that the spin- $(S, s)$ ferrimagnetic low-temperature expansions are obtained by replacing $J$ and $s$ by $-J$ and $-s$, respectively, in Eqs. (4.8) and (4.9). In other words, antiferromagnetically coupled alternating spins $S$ and $s$ look like a ferromagnetic assembly of virtual spins $[S s(S-s) / 2]^{1 / 3}=\left[(S+s)^{2} /(S-s)^{2}\right.$ $-1]^{1 / 3}(S-s) / 2$ at low temperatures. The quantity $[S s(S$ $-s) / 2]^{1 / 3}$ is less intuitive than the corresponding $[S s(S$ $+s) / 2]^{1 / 3}$ in the case of ferromagnetic coupling, but it results in a realistic spin quantum number $S-s$ when $S$ is equal to $2 s$. Equation (4.8) is nothing but the susceptibility of the spin- $(S, s)$ ferrimagnetic chain if we set $\tilde{t}$ for $k_{B} T / J S s(S-s)$ instead of $k_{B} T / J S s(S+s)$. All in all, the low-temperature physics is scaled by $S+s$ in ferromagnetic chains, whereas by $S-s$ in ferrimagnetic chains.

\section{CONCLUDING REMARKS}

Thermodynamic properties of alternating-spin Heisenberg ferromagnetic chains have been investigated in comparison with heterospin ferrimagnetic and homospin ferromagnetic chains. The magnetic susceptibility is a monotonically decreasing function of temperature regardless of $(S, s)$ and is qualitatively the same as those of uniform ferromagnetic chains. The specific heat qualitatively varies with $(S, s)$ and exhibits a rich structure at intermediate temperatures. It may be double-peaked for $S \gtrsim 3 s$ in general.

The low-temperature behavior has been revealed analytically. The thermal quantities are still expanded in powers of $T^{1 / 2}$ and exhibit ferromagnetic features. The conventional spin-wave theory misunderstands the low-temperature behavior as series of $T$. The missing terms are reproduced through the modified procedure. Ferromagnetic and ferrimagnetic mixed-spin chains are qualitatively alike at low temperatures. The spin- $(S, s)$ ferromagnetic chain looks similar to a ferromagnetic assembly of virtual spins $[S s(S$ $+s) / 2]^{1 / 3}=\left[1-(S-s)^{2} /(S+s)^{2}\right]^{1 / 3}(S+s) / 2 \simeq(S+s) / 2$, while the spin- $(S, s)$ ferrimagnetic chain behaves similar to that of virtual spins $[S s(S-s) / 2]^{1 / 3}=\left[(S+s)^{2} /(S-s)^{2}-1\right]^{1 / 3}(S-s) / 2$. The present findings are really complementary to the sophisticated high-temperature series-expansion calculations. ${ }^{39,40}$

The existent bimetallic chain ferromagnet $\mathrm{MnNi}\left(\mathrm{NO}_{2}\right)_{4}(\mathrm{en})_{2}$ possesses a rather weak intrachain exchange coupling $\left(J / k_{B} \simeq 2 \mathrm{~K}\right)$, in which the low-temperature thermodynamics revealed here is hard to verify. Nevertheless such a pioneering material must highly motivate further explorations in both chemical and physical fields, as was the case with uniform ferromagnetic chains. ${ }^{30-32,41-43}$ In addition to bimetallic chain compounds, several authors ${ }^{44}$ made a novel attempt to design low-dimensional heterospin systems utilizing organic triradicals. Mixed-spin chains contain further interesting topics such as dynamic structure factors of dual aspect ${ }^{45}$ and nuclear spin relaxation through the exchange-scattering-enhanced three-magnon process. ${ }^{46} \mathrm{We}$ hope our study will stimulate further experimental investigations into mixed-spin chain compounds.

\section{ACKNOWLEDGMENTS}

The authors are grateful to N. Fukushima for valuable comments. This work was supported by the Ministry of Education, Culture, Sports, Science and Technology of Japan, and the Iketani Science and Technology Foundation.
${ }^{1}$ O. Kahn, Y. Pei, and Y. Journaux, in Inorganic Materials, edited by D. W. Bruce and D. O'Hare (Wiley, New York, 1992), p. 95.

${ }^{2}$ A. Gleizes and M. Verdaguer, J. Am. Chem. Soc. 103, 7373 (1981); 106, 3727 (1984).

${ }^{3}$ O. Kahn, Y. Pei, M. Verdaguer, J.-P. Renard, and J. Sletten, J. Am. Chem. Soc. 110, 782 (1988); P. J. van Koningsbruggen, O. Kahn, K. Nakatani, Y. Pei, J.-P. Renard, M. Drillon, and P. Legoll, Inorg. Chem. 29, 3325 (1990).

${ }^{4}$ Y. Pei, M. Verdaguer, O. Kahn, J. Sletten, and J.-P. Renard, J. Am. Chem. Soc. 108, 7428 (1986).

${ }^{5}$ A. Caneschi, D. Gatteschi, P. Rey, and R. Sessoli, Inorg. Chem. 27, 1756 (1988); A. Caneschi, D. Gatteschi, J.-P. Renard, P. Rey, and R. Sessoli, ibid. 28, 1976 (1989); 28, 2940 (1989).

${ }^{6}$ A. S. Ovchinnikov, I. G. Bostrem, V. E. Sinitsyn, A. S. Boyarchenkov, N. V. Baranov, and K. Inoue, J. Phys.: Condens. Matter 14, 8067 (2002).

${ }^{7}$ M. Drillon, J. C. Gianduzzo, and R. Georges, Phys. Lett. 96A,
413 (1983); M. Drillon, E. Coronado, R. Georges, J. C. Gianduzzo, and J. Curely, Phys. Rev. B 40, 10992 (1989).

${ }^{8}$ A. K. Kolezhuk, H.-J. Mikeska, and S. Yamamoto, Phys. Rev. B 55, R3336 (1997).

${ }^{9}$ S. Brehmer, H.-J. Mikeska, and S. Yamamoto, J. Phys.: Condens. Matter 9, 3921 (1997).

${ }^{10}$ S. K. Pati, S. Ramasesha, and D. Sen, Phys. Rev. B 55, 8894 (1997); J. Phys.: Condens. Matter 9, 8707 (1997).

${ }^{11}$ T. Fukui and N. Kawakami, Phys. Rev. B 55, R14709 (1997); 56, 8799 (1997).

${ }^{12}$ G.-S. Tian, Phys. Rev. B 56, 5355 (1997).

${ }^{13}$ H. Niggemann, G. Uimin, and J. Zittartz, J. Phys.: Condens. Matter 9, 9031 (1997); 10, 5217 (1998).

${ }^{14}$ T. Sakai and S. Yamamoto, Phys. Rev. B 60, 4053 (1999); S. Yamamoto and T. Sakai, ibid. 62, 3795 (2000).

${ }^{15}$ N. B. Ivanov, Phys. Rev. B 62, 3271 (2000).

${ }^{16}$ O. Kahn, E. Bakalbassis, C. Mathonière, M. Hagiwara, K. Kat- 
sumata, and L. Ouahab, Inorg. Chem. 36, 1530 (1997).

${ }^{17}$ R. Feyerherm, C. Mathonière, and O. Kahn, J. Phys.: Condens. Matter 13, 2639 (2001).

${ }^{18}$ B. Gillon, C. Mathonière, E. Ruiz, S. Alvarez, A. Cousson, T. M. Rajendiran, and O. Kahn, J. Am. Chem. Soc. 124, 14433 (2002).

${ }^{19}$ N. Fukushima, A. Honecker, S. Wessel, and W. Brenig, Phys. Rev. B 69, 174430 (2004).

${ }^{20}$ F. Lloret, R. Ruiz, M. Julve, J. Faus, Y. Journaux, I. Castro, and M. Verdaguer, Chem. Mater. 4, 1150 (1992).

${ }^{21}$ J. Larionova, O. Kahn, J. Bartolome, R. Burriel, M. Castro, V. Ksenofontov, and P. Gütlich, Chem. Mater. 11, 3400 (1999).

${ }^{22}$ S. Yamamoto, Phys. Rev. B 69, 064426 (2004).

${ }^{23}$ S. Yamamoto, T. Fukui, K. Maisinger, and U. Schollwöck, J. Phys.: Condens. Matter 10, 11033 (1998).

${ }^{24}$ S. Yamamoto and T. Fukui, Phys. Rev. B 57, R14008 (1998).

${ }^{25}$ F. Bloch, Z. Phys. 61, 206 (1930); 74, 295 (1932).

${ }^{26}$ T. Holstein and H. Primakoff, Phys. Rev. 58, 1098 (1940).

${ }^{27}$ F. J. Dyson, Phys. Rev. 102, 1217 (1956); 102, 1230 (1956).

${ }^{28}$ S. Yamamoto, S. Brehmer, and H.-J. Mikeska, Phys. Rev. B 57, 13610 (1998).

${ }^{29}$ N. B. Ivanov, Phys. Rev. B 57, R14024 (1998); N. B. Ivanov, J. Richter, and U. Schollwöck, ibid. 58, 14456 (1998).

${ }^{30}$ M. Takahashi, Prog. Theor. Phys. Suppl. 87, 233 (1986).
${ }^{31}$ M. Takahashi and M. Yamada, J. Phys. Soc. Jpn. 54, 2808 (1985); M. Yamada and M. Takahashi, ibid. 55, 2024 (1986).

${ }^{32}$ M. Takahashi, Phys. Rev. Lett. 58, 168 (1987).

${ }^{33}$ S. Yamamoto, T. Fukui, and T. Sakai, Eur. Phys. J. B 15, 211 (2000).

${ }^{34}$ N. Fukushima, A. Honecker, S. Wessel, S. Grossjohann, and W. Brenig, Physica B 359-361, 1409 (2005).

${ }^{35}$ A. W. Sandvik, Phys. Rev. B 59, R14157 (1999).

${ }^{36}$ S. Yamamoto, Phys. Rev. B 59, 1024 (1999).

${ }^{37}$ T. Nakanishi and S. Yamamoto, Phys. Rev. B 65, 214418 (2002).

${ }^{38}$ S. V. Maleev, Sov. Phys. JETP 6, 776 (1958).

${ }^{39}$ O. Rojas, S. M. de Souza, and M. T. Thomaz, J. Math. Phys. 43, 1390 (2002).

${ }^{40}$ N. Fukushima, J. Stat. Phys. 111, 1049 (2003).

${ }^{41}$ C. P. Landee and R. D. Willett, Phys. Rev. Lett. 43, 463 (1979).

${ }^{42}$ P. Schlottmann, Phys. Rev. Lett. 54, 2131 (1985).

${ }^{43}$ M. Takahashi, P. Turek, Y. Nakazawa, M. Tamura, K. Nozawa, D. Shiomi, M. Ishikawa, and M. Kinoshita, Phys. Rev. Lett. 67, 746 (1991).

${ }^{44}$ Y. Hosokoshi, K. Katoh, Y. Nakazawa, H. Nakano, and K. Inoue, J. Am. Chem. Soc. 123, 7921 (2001).

${ }^{45}$ S. Yamamoto and T. Sakai, J. Phys. Soc. Jpn. 67, 3711 (1998).

${ }^{46}$ H. Hori and S. Yamamoto, J. Phys. Soc. Jpn. 73, 1453 (2004); J. Phys.: Condens. Matter 16, 9023 (2004). 